\title{
Hemophagocytic Lymphohistiocytosis after Lung Transplantation
}

\author{
Ah Young Leem, M.D., Sung Woo Moon, M.D., Song Yee Kim, M.D., Moo Suk Park, M.D., Young Sam Kim, M.D., \\ Se Kyu Kim, M.D., Joon Chang, M.D., Hyo Chae Paik, M.D., June Won Cheong, M.D. , \\ and Kyung Soo Chung, M.D.
}

Division of Pulmonology, Department of Internal Medicine, *Department of Thoracic and Cardiovascular Surgery, 'Division of Hematology, Department of Internal Medicine, Institute of Chest Disease, Severance Hospital, Yonsei University College of Medicine, Seoul, Korea

\begin{abstract}
Hemophagocytic lymphohistiocytosis $(\mathrm{HLH})$ is a rare but fatal complication after solid organ transplantation. Acquired forms of $\mathrm{HLH}$ are described in association with severe sepsis, autoimmune disorders, malignancy, immune-compromised states, infections, and solid organ transplantation. We experienced a case of hemophagocytic lymphohistiocytosis after bilateral lung transplantation. Leukopenia, thrombocytopenia, and hyperbilirubinemia were noted and became aggravated 50 days after transplantation. Diagnosis of HLH was based on clinical and laboratory findings of splenomegaly, cytopenia, elevated ferritin, elevated interleukin-2 receptor, and hemophagocytosis in bone marrow. Other features such as elevated bilirubin, lactate dehydrogenase, and D-dimer which can be present in HLH were also noted. The patient was immediately treated with etoposide and dexamethasone. Despite aggressive therapy, the patient deteriorated and died. Awareness of the diagnostic criteria of HLH after lung transplantation is important for clinicians.
\end{abstract}

Key Words: hemophagocytic; lung transplantation; lymphohistiocytosis.

Hemophagocytic lymphohistiocytosis (HLH), also known as hemophagocytic syndrome, is a rare and fatal disease that may occur in solid organ transplant recipients.[1] This syndrome is characterized by phagocytizing histiocytes that infiltrate tissues and phagocytize blood elements and their precursors.[1,2] Genetic forms of HLH are associated with genetic defects in the function of natural killer (NK) cells and cytotoxic T cells. Acquired forms of HLH are described in association with severe sepsis, autoimmune disorders, malignancy, immune-compromised states, infections, and solid organ transplantation.[3] Despite the use of the therapy such as etoposide, cyclosporine, and steroids, the prognosis of this syndrome remains poor.[1,3]

Some cases of reactive HLH after solid organ transplantation have been reported; however, most of the cases were associated with kidney transplantation. Only a few cases after lung transplantation have been reported.[2,4]

Herein, we report a case of severe HLH after bilateral lung transplantation.

Received on November 26, 2014 Revised on January 3, 2015

Accepted on January 13, 2015

Correspondence to: Kyung Soo Chung, Division of Pulmonology, Department of Internal Medicine, Institute of Chest Disease, Severance Hospital, Yonsei University College of Medicine, 50-1 Yonsei-ro, Seodaemun-gu, Seoul 120-752, Korea

Tel: +82-2-2227-4203, Fax: +82- 2-393-6884

E-mail: chungks@yuhs.ac

*No potential conflict of interest relevant to this article was reported.

\section{Case Report}

A 60-year-old man who was waiting lung transplantation was admitted for aggravation of idiopathic pulmonary fibrosis. Previous pulmonary function test showed forced vital capacity $32 \%$ of predicted value, forced expiratory volume in one second $35 \%$ of predicted value, total lung capacity $42 \%$

(cc) This is an Open Access article distributed under the terms of the Creative Commons Attribution Non-Commercial License (http://creativecommons.org/ licenses/by-nc/3.0/) which permits unrestricted non-commercial use, distribution, and reproduction in any medium, provided the original work is properly cited. Copyright (c) 2015 The Korean Society of Critical Care Medicine 
of predicted value and lung diffusing capacity for CO 30\% of predicted value. Despite of treatment, he was worsened and in need of ventilator support. However he could not undergo bilateral lung transplantation until 2 weeks after intubation.

On postoperative day (POD) 25, leukopenia and thrombocytopenia were noted and became aggravated. On POD 33 , fever spiking was noted and multifocal ground glass opacities and consolidations were demonstrated on chest computed tomography. Acute rejection was suspected and steroid pulse therapy was performed. After the therapy, the chest X-ray result improved, and he was transferred to the general ward from the intensive care unit (ICU). His general condition was gradually improving.

However, on POD 57, he complained of low-grade fever, intermittent chest discomfort, and dyspnea. Echocardiography revealed stress-induced cardiomyopathy. He was readmitted to the ICU again due to septic and cardiogenic shock. Shortly after, he was infected with multidrug resistant Acinetobacter baumannii pneumonia and treated with antibiotics (carbapenem, vancomycin, and colistin). Despite of the treatment he was worsened, and antifungal agent was added. Serum bilirubin had started to rise (total bilirubin 2.1 $\mathrm{mg} / \mathrm{dL}$ ) from POD 50 and had gradually increased (total bilirubin $39.6 \mathrm{mg} / \mathrm{dL}$, direct bilirubin $30.7 \mathrm{mg} / \mathrm{dL}$ ) by POD 80 . An abdominal ultrasound demonstrated splenomegaly (11.4 $\mathrm{cm}$ ) and a moderate degree of ascites yet no evidence of hepatomegaly, intrahepatic duct dilatation, or common bile duct dilatation. Acute renal failure was developed and aggravated. Continuous renal replacement therapy was applied since POD 68. Pancytopenia was also aggravated (white blood cell $1,700 / \mu \mathrm{L}$, and neutrophils $1,326 / \mu \mathrm{L}$, hemoglobin $9.0 \mathrm{~g} / \mathrm{dL}$, platelets $\left.46 \times 10^{3} / \mu \mathrm{L}\right)$ (Fig. 1). Fibrinogen (421 $\mathrm{mg} / \mathrm{dL}$; normal range: $200-400 \mathrm{mg} / \mathrm{dL})$, ferritin $(4,518 \mathrm{ng} /$ $\mathrm{mL}$; normal range: $23.9-336.2 \mathrm{ng} / \mathrm{mL}$ ), and soluble interleukin-2 receptor $(8,730 \mathrm{U} / \mathrm{mL}$; normal range: $124-466 \mathrm{U} / \mathrm{mL})$ were also elevated. The level of triglyceride was normal (127 $\mathrm{mg} / \mathrm{dL}$, normal range: $48-200 \mathrm{mg} / \mathrm{dL}$ ). Neurological abnormalities or rash were not observed. In the early postoperative period, cytopenia was thought to be related to the use of broad-spectrum antibiotics and immunosuppressive drugs. Because the cause of ongoing pancytopenia was unclear, we conducted bone marrow aspiration and biopsy on POD 82, and histiocytes were frequently seen with occasional hemo-
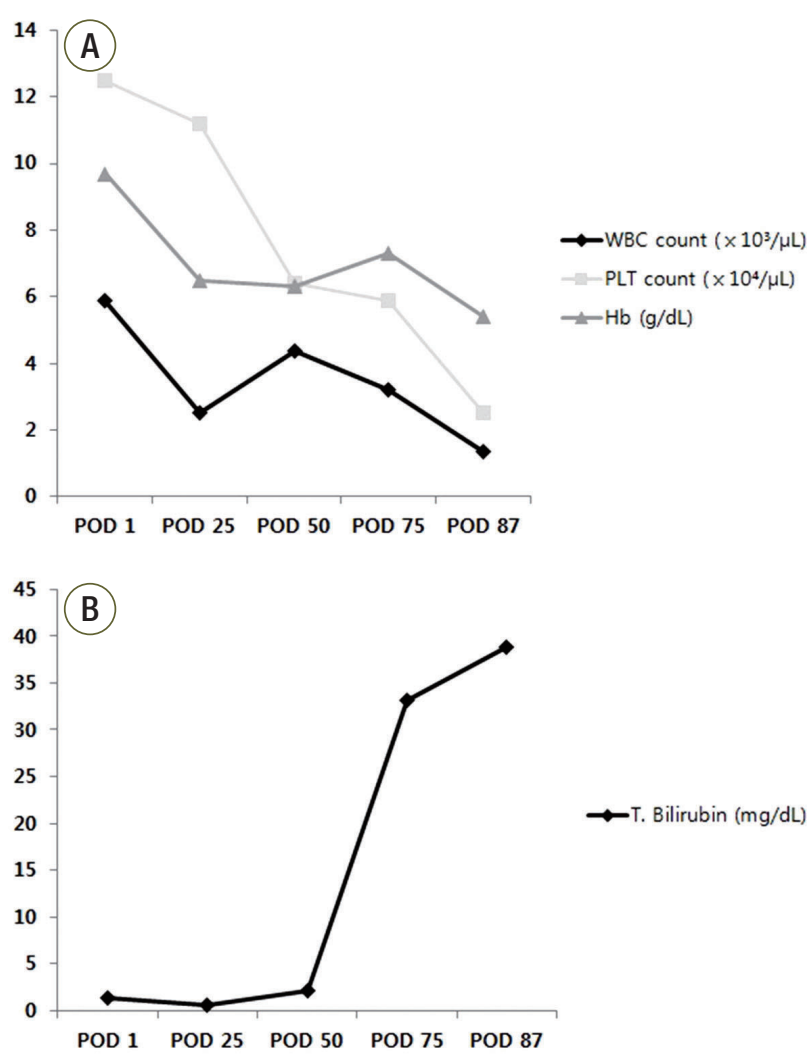

Fig. 1. Complete blood cell count (Fig. 1A) and total bilirubin (Fig. 1B). Pancytopenia and hyperbilirubinemia became aggravated after lung transplantation. WBC: white blood cell; PLT: platelet; $\mathrm{Hb}$ : hemoglobin; POD: postoperative day; T. bilirubin: total bilirubin.

phagocytes (Fig. 2). HLH was diagnosed based on the clinical and laboratory findings, together with the bone marrow biopsy results. He was treated immediately with $75 \mathrm{mg} / \mathrm{m}^{2}$ of etoposide ( $50 \%$ dose reduction due to renal and hepatic failure for one day) and $10 \mathrm{mg} / \mathrm{m}^{2}$ of dexamethasone (for 3 days). Four days after, despite aggressive therapy, his condition severely deteriorated, and he died from vancomycinresistant Enterococcus faecium sepsis on POD 87.

\section{Discussion}

HLH is an aggressive and potentially fatal hyper-inflammatory syndrome. It results from inappropriate activation of lymphocytes and macrophages. Genetic forms of HLH are associated with genetic defects in the function of NK cells and cytotoxic T cells. Acquired forms of HLH are 


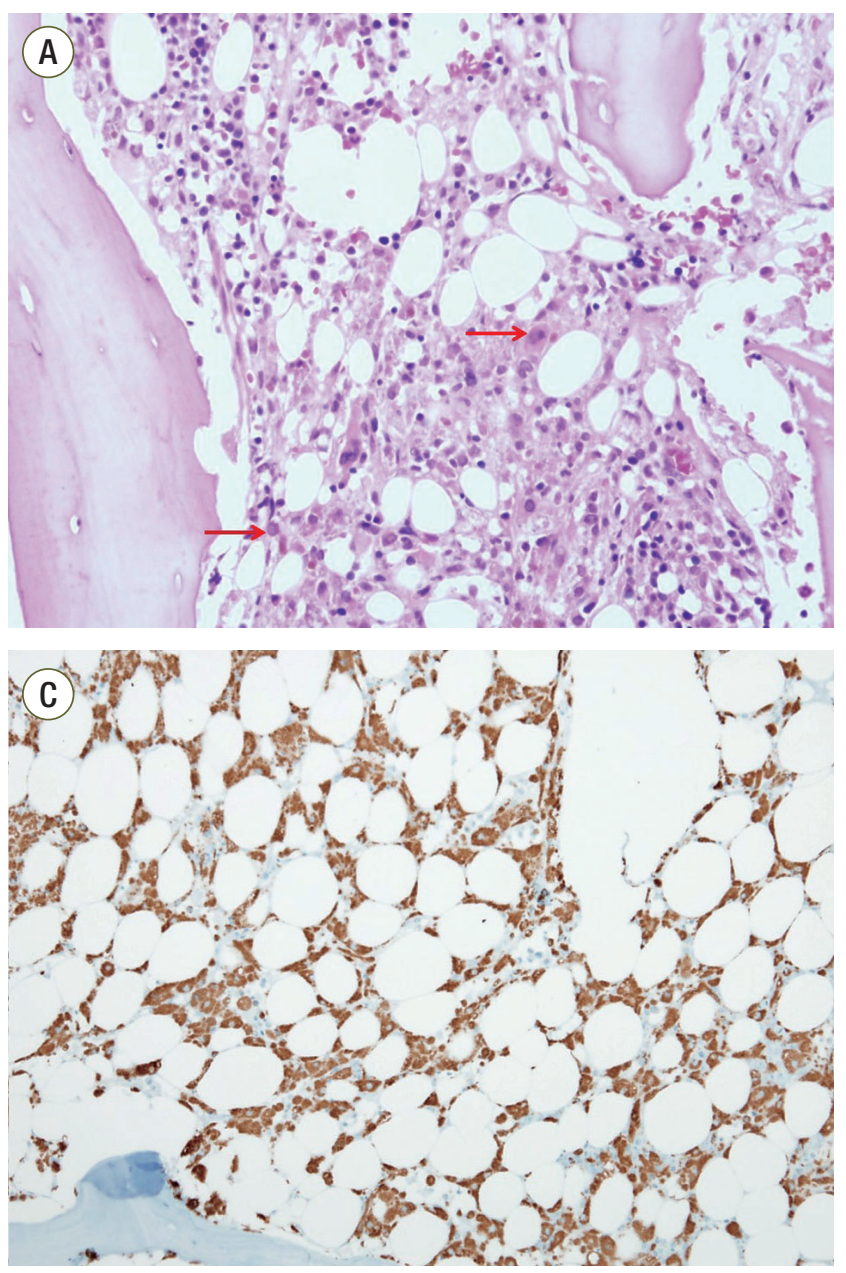

caused by infection or other stimuli such as autoimmune diseases, malignancies, and acquired immune deficiencies. This syndrome is caused from widespread tissue infiltration by phagocytizing histiocytes with direct cytotoxicity of activated macrophages that infiltrate tissues and phagocytize blood elements and their precursors.[1]

According to the diagnostic guidelines of HLH-2004, a diagnosis of HLH can be made with at least five of eight criteria: (1) fever, (2) splenomegaly, (3) cytopenia of two or more cell lines (hemoglobin, platelets, and neutrophils), (4) hypertriglyceridemia or hypofibrinogenemia, (5) elevated ferritin, (6) elevated interleukin-2 receptor, (7) decreased natural killer cell activity, and (8) hemophagocytosis in bone marrow.[1] Current treatment regimens usually involve high dose corticosteroids, etoposide and cyclosporine.[1] Despite aggressive therapy, HLH is associated with poor prognosis. $[1,3]$

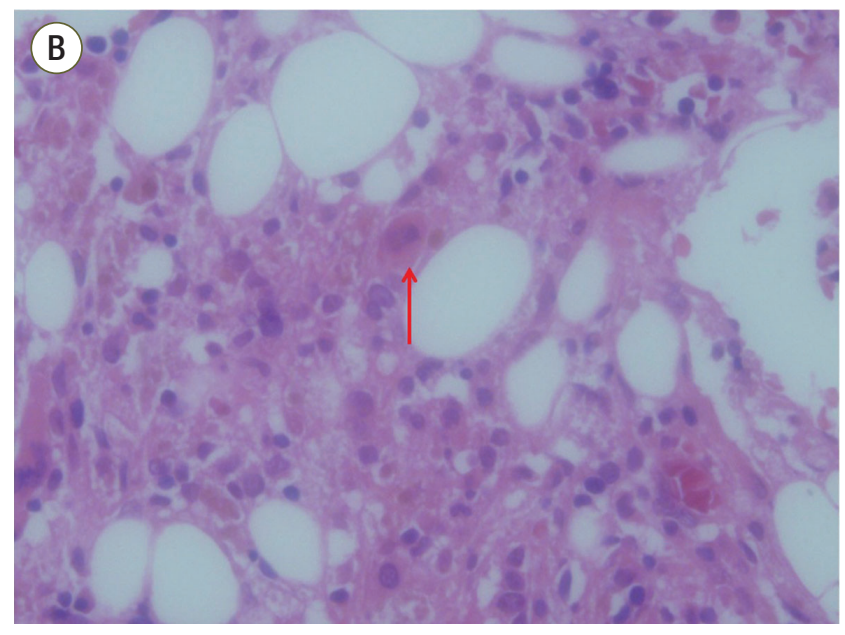

Fig. 2. Microscopic finding from a bone marrow biopsy specimen (Fig. 2A and Fig. 2B) and immunohistochemical staining for CD68 (Fig. 2C). The microscopic finding from the bone marrow biopsy specimen shows prominent hemosiderin deposition and multifocal fat necrosis with foamy macrophage infiltrates (Fig. 2A [x100] and Fig. 2B [X400]). Immunohistochemical staining for CD68 shows an increased number of stromal macrophages (Fig. 2C).

Some cases of reactive HLH after solid organ transplantation have been reported. The first series of transplant patients affected with HLH were described by Risdall et al. in 1979, who reported that HLH was associated with viral infections in 19 patients (including 13 kidney transplant recipients).[5] Karras et al. reported that the incidence of HLH after kidney transplantation was $0.4 \%$ with a mortality of $47 \%$.[6] However, only a few cases of HLH after lung transplantation have been reported. Oto et al[2] reported a case of severe HLH related to Pseudomonas infection in a single lobar lung transplant recipient. Diaz-Guzman et al[4] reported two patients who developed hemophagocytic lymphohistiocytosis after having a lung transplant and presented a review of all cases of hemophagocytic lymphohistiocytosis occurring in solid organ transplant recipients. No cause was found in the first case, and Epstein-Barr virus was implicated as the causative agent in the second one. 
In this case, a diagnosis of HLH was based on clinical and laboratory findings of splenomegaly, cytopenia, elevated ferritin, elevated interleukin-2 receptor, and hemophagocytosis in bone marrow. Other features such as elevated bilirubin, LDH, and D-dimer, which can be present in HLH, were also noted. Hyperfibrinogenemia was thought to be related to a disseminated intravascular coagulation because elevated D-dimer, prothrombine time, and activated partial thromboplastin time were also noted.

HLH and sepsis share high levels of pro- and anti-inflammatory molecules and many laboratory findings such as cytopenia, elevated ferritin, and hyperbilirubinemia. In fact, severe sepsis with multiple organ failure was noted in this case finally. However, hemophagocytosis in bone marrow was a useful finding for the diagnosis of HLH.

Opportunistic infections associated with immunocompromised status can be the cause of HLH. In patients with defined infections, trigger-directed therapy is recommended. However, according to HLH-2004 protocol, HLH-specific chemotherapy is recommended when HLH is highly suspected, regardless of the forms of HLH (genetic forms or acquired forms).[3] So, in our case, concomitant HLHdirected standard treatment was been administered.

In conclusion, HLH is an uncommon yet potentially lethal complication that may develop in solid organ transplant patients, including lung transplant recipients. Clinical presentation is nonspecific, and patients may present with unexplained sepsis or multiple organ failure. Therefore, clinicians should consider HLH as a possible diagnosis in the clinical context after lung transplantation.

\section{References}

1) Janka GE, Lehmberg K: Hemophagocytic syndromesan update. Blood Rev 2014; 28: 135-42.

2) Oto T, Snell GI, Goto K, Miyoshi S: Hemophagocytic syndrome: a rare but specific complication of lung transplantation. J Thorac Cardiovasc Surg 2010; 140: e58-9.

3) Henter JI, Horne A, Aricó M, Egeler RM, Filipovich AH, Imashuku S, et al: HLH-2004: diagnostic and therapeutic guidelines for hemophagocytic lymphohistiocytosis. Pediatr Blood Cancer 2007; 48: 124-31.

4) Diaz-Guzman E, Dong B, Hobbs SB, Kesler MV, Hayes D Jr: Hemophagocytic lymphohistiocytosis after lung transplant: report of 2 cases and a literature review. Exp Clin Transplant 2011; 9: 217-22.

5) Risdall RJ, McKenna RW, Nesbit ME, Krivit W, Balfour HH Jr, Simmons RL, et al: Virus-associated hemophagocytic syndrome: a benign histiocytic proliferation distinct from malignant histiocytosis. Cancer 1979; 44: 993-1002.

6) Karras A, Thervet E, Legendre C; Groupe Coopératif de transplantation d'lle de France: Hemophagocytic syndrome in renal transplant recipients: report of 17 cases and review of literature. Transplantation 2004; 77: 238-43. 